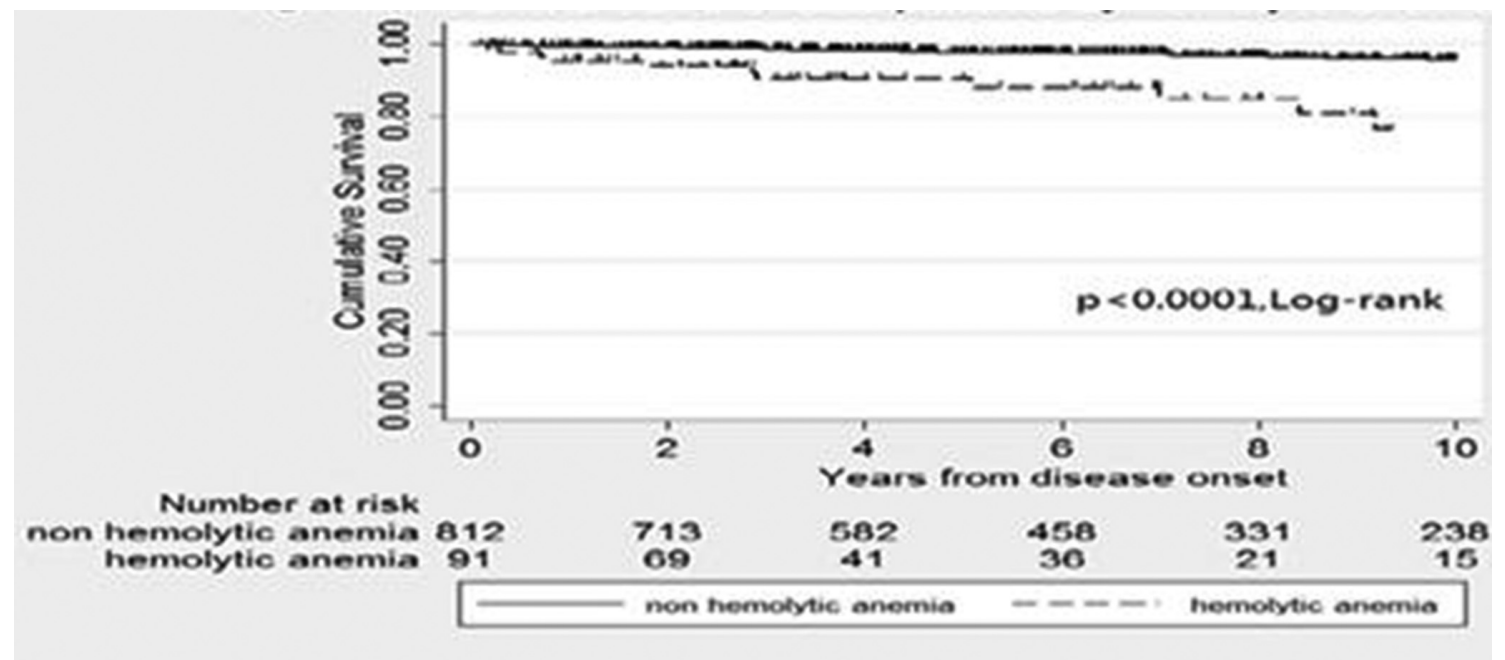

Abstract 412 Figure 3 Survival curve of SLE patients by hemolytic anemia.

\section{LUPUS NEPHRITIS IN INDIGENOUS AUSTRALIANS: INCIDENCE, HISTOLOGY AND OUTCOME}

1J Nossent*, ${ }^{2} \mathrm{~F}$ Ghazanfari, ${ }^{3} \mathrm{Z}$ Jabbar. ${ }^{1}$ The University of Western Australia, School of Medicine, Perth, Australia; ${ }^{2}$ Maroondah Consulting, Rheumatology, Melbourne, Australia; ${ }^{3}$ Royal Darwn Hospital, Nephrology, Darwin, Australia

\subsection{6/lupus-2017-000215.413}

Background and aims Ethnicity impacts on disease frequency and severity in patients with Lupus Nephritis (LN). As such data are lacking for Indigenous Australians (IA), we investigated the epidemiology, histopathology and outcomes of biopsy proven LN in the Top End of the Northern Territory, Australia.

Methods All patients with biopsy confirmed LN between 2001 and January 2011 were identified from a central database at the Royal Darwin Hospital. Demographics and renal histopathology at first renal biopsy (ISN classification, NIH activity (AI) and chronicity index (CI), immunofluorescence (IF) and electron dense deposit (EDD)) were recorded. Clinical findings and outcomes were retrieved by chart review.

Results The total study cohort $(n=42$, age 30 years; $86 \%$ female) included 31 IA patents (74\%). The annual LN incidence was $7 / 100,000$ in IA patients versus $0.7 / 100,000$ in non-indigenous $(\mathrm{NI})$ patients $(\mathrm{p}<0.01)$.

Proliferative LN (class III+IV) was less frequent in IA patients $(42 \%$ vs. $72 \%) \quad(\mathrm{p}<0.01)$, but AI $(2$ vs. 5, $\mathrm{p}=0.06)$ and CI ( 2 vs. $1, \mathrm{p}=0.9)$ scores did not differ significantly.

Five and ten year patient survival rates were $69 \%$ and $50 \%$ for IA and $90 \%$ for NI patients, while renal survival was $87 \%$ and $53 \%$ in IA patients and $100 \%$ in NI patients. The leading causes of death were infections (38.6\%), end stage renal disease (23\%), cardiovascular events (15.4\%).

Conclusions Biopsy proven LN occurs much more frequently in the IA than in the Causcasion population. While associated with poor patient and renal survival, this is not explained by the histological severity of LN.

\section{PREDICTORS OF END-STAGE RENAL DISEASE IN FILIPINOS WITH LUPUS NEPHRITIS: A CASE-CONTROL STUDY}

B Paras*, E Venegas, S Navarra. University of Santo Tomas Hospital, Internal Medicine, Manila, Philippines

\subsection{6/lupus-2017-000215.414}

Background and aims End-stage renal disease (ESRD) is a major cause of morbidity and mortality in systemic lupus erythematosus (SLE). We studied the risk factors for development of ESRD among Filipino patients with lupus nephritis.

Methods Included were ESRD patients entered in the SLE database of University of Santo Tomas (UST) Hospital, Manila, Philippines from 2005 to 2015. The control group consisted of lupus nephritis patients without ESRD from same database - matched for age, gender and disease duration. Associations of risk factors including comorbidities and treatment for ESRD were tested using Cox-proportional hazard model.

Results Cases included 71 ESRD patients $(58,81.7 \%$ females,

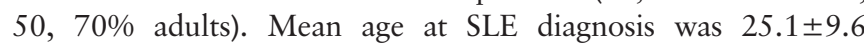
(7-47) years, mean SLE disease duration 9.5 \pm 5.5 (1-29) years, and mean disease duration from SLE diagnosis to ESRD $5.0 \pm 3.0(0.5-14)$ years. By multivariate analysis, hypertension [hazard ratio (HR) 3.754; 95\% CI 2.230, 6.320; p <0.001], and diabetes mellitus (HR 3.775; 95\% CI 1.691, 8.425; $\mathrm{p}<0.001)$ prior to SLE diagnosis were independently associated with progression to ESRD. Onset and treatment of nephritis, and antiphospholipid antibody syndrome (APS) did not significantly impact progression to ESRD. Patients with both hypertension and APS had the shortest disease duration prior to ESRD, but this did not achieve statistical significance.

Conclusions We showed that hypertension and diabetes mellitus prior to SLE diagnosis were strongly associated with progression to ESRD in SLE, reinforcing need to recognise and aggressively control these co-morbidities early at SLE diagnosis, including minimal use of aggravating factors like corticosteroids. 